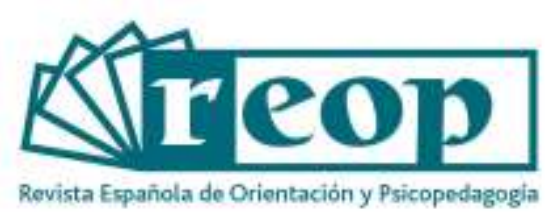

\title{
EVOLUCIÓN E INCIDENCIA, EN WOS, DE LA DIVERSIDAD EN EL ÁMBITO EDUCATIVO
}

\section{EVOLUTION AND INCIDENCE, IN WOS, OF DIVERSITY IN THE FIELD OF EDUCATION}

\author{
Antonio-José Moreno-Guerrero ${ }^{1}$ \\ Universidad de Granada. Facultad de Educación, Economía y Tecnología. Departamento de \\ Didáctica y Organización Escolar. Ceuta, España. \\ María Angustias Ortiz Molina \\ Departamento de Didáctica y Organización Escolar. Ceuta, España. \\ Fernando-José Sadio-Ramos \\ Departamento de Didáctica y Organización Escolar. Ceuta, España. Escolar Superior de \\ Educacao. Instituto Politécnico de Coimbra. Coimbra, Portugal. \\ Santiago Alonso-García \\ Departamento de Didáctica y Organización Escolar. Ceuta, España.
}

\section{RESUMEN}

La diversidad en el ámbito educativo abarca una gran cantidad de acepciones, tales como la diversidad cultural, lingüística, de identidad sexual, de género y de capacidad educativa, entre otros aspectos. La finalidad de este estudio es analizar el desarrollo de las investigaciones sobre la diversidad en el ámbito educativo, teniendo en cuenta la producción científica en la base de datos de Web of Science (WoS), en el periodo comprendido entre 2009 y 2019, ambos inclusive. Para ello se ha desarrollado un estudio bibliométrico haciendo uso de técnicas de rastreo, cuantificación documental y mapeo analítico. Se han analizado un total de 25958 documentos extraídos de la búsqueda, realizada en Web of Science, mediante las siguientes combinaciones de palabras: diversity AND education, diversity AND inclusión y diversity AND intervention. Los

\footnotetext{
${ }^{1}$ Correspondencia: Antonio-José Moreno-Guerrero. Correo-e: ajmoreno@ugr.es
} 
resultados muestran el auge de la temática de estudio en la comunidad educativa, en la que los artículos escritos en inglés son los principales medios utilizados para comunicar las investigaciones, siendo Estados Unidos el país que más repercusión tiene actualmente. Se puede concluir que la temática "diversidad" se centra en aspectos relacionados con la equidad, la justicia social, los estudiantes, la identidad, la inclusión, la educación, el multiculturalismo y el género, aunque también está cogiendo fuerza estudios relacionados con las actitudes, las mujeres y la desnutrición, siendo cada vez más relevantes y abundantes dichas investigaciones sobre esta temática.

Palabras clave: diversidad, intervención, bibliometría, equidad, inclusión.

\begin{abstract}
Diversity in education encompasses a wide range of meanings, such as cultural diversity, linguistic diversity, sexual identity, gender diversity and educational capacity, among others. The purpose of this study is to analyze the development of research on diversity in the educational field, taking into account the scientific production in the Web of Science (WoS) database, in the period from 2009 to 2019, both inclusive. To this end, a bibliometric study has been developed using tracking techniques, documentary quantification and analytical mapping. A total of 25958 documents extracted from the Web of Science search were analysed using the following word combinations: diversity AND education, diversity AND inclusion and diversity AND intervention. The results show the rise of the study topic in the educational community, in which articles written in English are the main means used to communicate the research, being the United States the country that has more repercussion at the moment. It can be concluded that the theme of diversity focuses on aspects related to equity, social justice, students, identity, inclusion, education, multiculturalism and gender, although studies related to attitudes, women and malnutrition are also gaining strength, with such research being increasingly relevant and abundant on this subject.
\end{abstract}

Key Words: diversity, intervention, bibliometrics, equity, inclusion.

\title{
Cómo citar este artículo:
}

Moreno-Guerrero, A.J., Ortiz-Molina, M.A. Sadio-Ramos, F.J., y Alonso-García, S. (2020). Evolución e incidencia, en WOS, de la diversidad en el ámbito educativo. Revista Española de Orientación y Psicopedagogía, 31(2), 56-77. https://doi.org/10.5944/reop.vol.31.num.2.2020.27986

\section{Introducción}

La diversidad en el ámbito educativo ofrece un campo muy amplio. Esta alberga diversas temáticas de estudios, ya sea diversidad cultural, discriminación o racismo (Leseth y Engelsrud, 2019), diversidad lingüística (Preece, 2019), por cuestiones de capacidad educativa (Azorín, 2017) e incluso diversidad sexual y de género (Airton y Koecher, 2019) en cualquier etapa educativa (Barbato y Turri, 2019). El término de diversidad suele ir asociado al de equidad (Byrd, 2019). Es decir, la diversidad, puede ser entendida como una característica intrínseca y extrínseca en los seres humanos. Es decir, intrínseca por las diferencias existentes entre las personas por la forma 
de pensar, sentir y actuar; y extrínseca por las diferencias socio-económicas y culturales que pueda existir (Jurado et al., 2020).

Muchos de los problemas que surgen con la diversidad, sea del tipo que sea, se centran principalmente en la visión que se tiene de la misma, dado que se concibe como una acción legal que debe tenerse presente y cumplirse, en vez de un valor de crecimiento y de rentabilidad para cualquier organización (Zhuwao et al., 2019). Además suele ser percibida como un deber, tanto de la escuela como del estado, con la intención de fomentar la atención a la diversidad, tratando de enriquecer a toda la comunidad educativa (Pegalajar, 2017).

En la normativa española, centrada en el ámbito educativo, la Ley Orgánica 2/2006, de 3 de mayo, de Educación, en su texto consolidado, tras su modificación parcial por la Ley Orgánica 8/2013, de 9 de diciembre, para la mejora de la calidad educativa (LOE-TC), hace referencia a la diversidad en el título II, denominado equidad en la Educación, centrándose tanto en el alumnado con necesidades específicas de apoyo educativo (NEAE). Esta definición incluye diversos tipos, entre los que se encuentran las necesidades educativas especiales (NEE), por dificultades específicas de aprendizaje (DEA), trastornos por déficit de atención e hiperactividad (TDAH), por sus altas capacidades intelectuales, por haberse incorporado tarde al sistema educativo; o por condiciones personales o de historia escolar; como a la compensación de las desigualdades en educación por factores sociales, económicos, culturales, geográficos, étnicos o de otra índole. Partiendo de la propia normativa, se indica que los principios en los que se enmarca la atención a la diversidad del alumnado son el de normalización e inclusión. Esto conlleva la necesidad de adaptar las enseñanzas a las necesidades del alumnado, no centradas únicamente en la propia diversidad funcional que presenten, sino también en las situaciones de desventaja socioeducativa.

Entre las actuaciones sobre la atención a la diversidad, se pueden encontrar diversos tipos de investigaciones sobre las temáticas anteriormente indicadas. Una de ellas es la educación inclusiva en la etapa de educación infantil, en la que la metodología requiere de cambios significativos, no centrados en los métodos expositivos, para que la enseñanza inclusiva sea real (De Araujo et al., 2019). Otras se centran en la red de Entornos y Métricas Inclusivas en Educación e Investigación, en el campo de la Biología (iEMBER, por sus siglas en inglés), la cual es una práctica educativa que involucra a los interesados en diversidad, equidad e inclusión. Esto se desarrolla mediante proyectos de colaboración interdisciplinarios, mediante la coordinación de esfuerzos a través de redes, desarrollo profesional e investigación colaborativa, generando con ello entornos inclusivos (Tennial et al., 2019).

En otro tipo de investigaciones la diversidad hace referencia al campo de la dietética, la cual debe atender a las barreras sistemáticas de estudiantes pertenecientes a minorías étnicas y bajos niveles socioeconómicos, con la intención de favorecer la equidad (Riediger et al., 2019). O también en las escuelas secundarias, donde se aborda la diversidad como un aspecto de la educación para la ciudadanía, en el que las prácticas de los profesores de secundaria, en relación con la enseñanza sobre la diversidad, se relacionan con los factores contextuales de la escuela, como la composición de los alumnos y la trayectoria educativa de los mismo, no siendo tratados de forma adecuada (Sincer et al., 2019).

El éxito de las actuaciones depende de las intervenciones educativas, las cuales deben llevarse a cabo desde una perspectiva integral, inclusiva y multidisciplinaria. Esto permite intervenir en actitudes, sentimientos, comportamientos sociales y agendas culturales a nivel individual, institucional y social (Veliz et al., 2019). En estos casos, tienen mucha importancia las emociones en la construcción de la ciudadanía, dado que los sentimientos de compasión y vulnerabilidad permiten conocer las circunstancia de los demás, promoviendo así una estrecha relación entre inteligencia emocional y comportamiento social (Sánchez y García, 2017). En el ámbito de la atención a la diversidad se centra en los entornos de aprendizaje (Amer y Mir, 2017). 
Además, la integración de las tecnologías de la información y comunicación (TIC) favorecen la atención a la diversidad, fomentando con ello la inclusión en los procesos formativos (Castro y Eirin, 2018).

Dada a la importancia que está tomando la diversidad en el ámbito educativo, se hace necesario realizar este estudio bibliométrico con la intención de ofrecer a la comunidad educativa las últimas tendencias en este campo de estudio. Por ello, a lo largo de la presente investigación se tratará de dar respuesta a las siguientes cuestiones: ¿En qué idiomas se divulgan mayormente la ciencia en este campo? ¿Cuáles son las organizaciones, fuentes de procedencia, autores y países más prolíficos en la investigación científica sobre la diversidad en el ámbito educativo? ¿Cuáles son las principales temáticas de investigación y qué tendencia tienen en los últimos diez años dentro de la propia comunidad educativa? ... Estas y otras cuestiones serán respondidas a lo largo del presente documento, dado que así se podrá establecer un perfil más claro y concreto del campo de estudio de la diversidad en el ámbito educativo. Además, las preguntas de investigación planteadas en esta investigación siguen los planteamientos ya formulados en otros estudios, de semejantes características, y de gran impacto científico (Hinojo-Lucena et al., 2020; Jurado et al., 2020).

\section{Método}

La finalidad de la presente investigación es estudiar el desarrollo de las investigaciones sobre la diversidad, en el ámbito educativo, de la producción científica en la base de datos de Web of Science, en el periodo comprendido entre 2009 y 2019, ambos inclusive. La elección de esta base de datos se debe a su relevancia en el ámbito internacional, siendo la principal base de datos de referencia, en lo que al número de citas se refiere, de Journal Citation Report (JCR). A su vez, la investigación trata de determinar la producción diacrónica de la temática de estudio, determinar la producción geográfica de los estudios en función al país e institución que firma el documento científico, analizar los autores con mayor producción científica en este campo de conocimiento, explorar la productividad de fuente en relación al tipo de publicación, título que la contiene, idioma, área de investigación y obras más citadas, identificar la continuidad de palabras clave entre los tres periodos establecidos (2009-2012, 2013-2016 y 2017-2019), comprobar la evolución científica sobre la diversidad en el ámbito educativo e identificar los temas motores de cada uno de los tiempos establecidos con anterioridad.

Con la intención de alcanzar las finalidades establecidas con anterioridad, el presente artículo se fundamenta en una metodología de tipo bibliométrico por revisión sistemática (Todeschini y Baccini, 2016; Rodríguez et al., 2019), haciendo uso de técnicas de rastreo analítico (Álvarez, 2015) y de cuantificación documental (Rodríguez et al., 2019) a través de una evaluación de rendimiento, en la que se analiza la producción científica de los actores científicos; y la creación de mapas de ciencia, valorando la evolución conceptual del objeto de estudio en un marco longitudinal (Cobo et al., 2011).

Este tipo de estudios sirven para determinar el estado actual de la situación de determinadas temáticas de estudio (Quevedo y López, 2010) como ocurre con la diversidad en el ámbito educativo, aspecto tratado en este documento. Los estudios bibliométricos de la producción científica sirven para ofrecer una visión de la difusión, siendo este tipo de análisis objetivos y verificables (Velasco et al., 2012), y un indicador de calidad para los nuevos estudios (Ruiz et al., 2010), a la vez que relevante para las revistas y los investigadores. Además, ofrece datos sobre la producción científica, su difusión y el impacto de las publicaciones y de los investigadores (Elkins 
et al., 2010; Franceschet, 2010), mostrando las últimas tendencias en las temáticas estudiadas y visualizando las líneas de investigación al alza (Velasco et al., 2012; López et al., 2015).

Los datos de estudios se han recopilado de la colección principal de Web of Science (febrerojunio 2019) haciendo uso de las unidades de análisis "diversity", "education", "inclusion" e "intervention", recopiladas de los tesauros de la UNESCO y ERIC. La ecuación de búsqueda se ha centrado en el tema, las palabras claves y los resúmenes de la producción científica, estableciéndose los siguientes operadores booleanos: "diversity" AND "education" (DIDE), "diversity" AND "inclusion" (DICL), y "diversity" AND "intervention" (INTE). Hay que destacar que todas las búsquedas se realizaron con el entrecomillado, para localizar las palabras exactas, y no palabras derivadas de las mismas. El total de documentos recopilados en todas las búsquedas realizadas son 25958, distribuidos de la siguiente manera: DIDE (15957), DICL (5871) e INTE (4130).

Para la evaluación de rendimiento y de producción científica se desarrolló un estudio descriptivo, analizando 9 variables siguiendo lo establecido en la matriz de protocolo PRISMA-P (Hutto et al., 2016): a) Año de publicación: 2009-2019; b) País: $x \geq 1100$; c) Institución: $x \geq 220$; d) Documentos por autor: $x \geq 13$; e) Tipo de fuente: $x \geq 580$; f) Idioma de fuente: $x \geq 110$; g) Área de investigación: $x \geq 1000 ;$ h) Impacto de fuentes (citas): $x \geq 100$.

Para el desarrollo estructural y dinámico del concepto de la diversidad en el ámbito educativo se ha desarrollado un análisis de co-palabras (Hirsch, 2005), fundamentado en el índice-h y en el número de citas (Cobo et al., 2011), generando un mapa de ciencia y un análisis de rendimiento para identificar y representar los subdominios conceptuales del campo de investigación y su progreso en la temática de estudio. El análisis de co-palabras longitudinal, establecido con el programa SciMAT (Cobo et al., 2012) se estructuró en cuatro fases (Montero et al., 2018):

- Detección de temas de investigación: De las 25958 referencias usadas anteriormente, se ha realizado un mapeo eliminando aquellas que no tienen palabras clave, los documentos duplicados y los que no tienen relación con la temática de investigación, quedándose en este caso con un total de 22230 referencias. Con estas referencias se ha creado una red de co-ocurrencia mediante nodos. La base son las palabras claves localizadas previamente, las cuales se conectan entre sí cuando dos de estas palabras co-aparecen en diversos textos científicos, generando una red normalizada de co-palabras. Mediante el algoritmo de clustering, se han localizado los temas de investigación, mostrándose así las palabras clave fuertemente relacionadas. Se usaron las palabras claves dadas por los autores y las creadas por WoS, según el tipo de documento. Además, se analizó toda la producción científica para comprobar que no hubiera documentos repetidos, y determinados documentos que aparecieron en la búsqueda que no eran propiamente de la temática tratada, haciendo uso del programa IBM SPSS Statistics v.20, el cual, mediante las tablas frecuencias, localiza los documentos repetidos.

- Representación tema investigación: Para ello se ha usado un diagrama estratégico y una red temática (Callon et al., 1991), formada por dos dimensiones (centralidad y densidad), donde las palabras clave se muestran en cuatro sectores: sector superior derecho, donde alberga los temas motores y fundamentales en la temática de investigación; sector superior izquierdo, donde las conexiones son débiles y son nodos con poca relevancia en la temática; sector inferior izquierdo, donde los temas son relevantes pero no tienen un desarrollo acuciado; y sector inferior derecho, donde los nodos carecen de desarrollo o relevancia, aunque los que aparecen en dicha zona pueden ser temas emergentes.

- Localización de áreas temáticas. Esto se determina por la evolución cronológica mostrada por los nodos de un periodo a otro. La fuerza de relación se fundamenta según el número de palabras clave que tienen en común. El análisis se inicia en 2009 hasta 2019. Los periodos establecidos han sido 2009-2012, 2013-2016 y 2017-2019. 
- Análisis de rendimiento. Cada una de las palabras clave, tienen a su vez, una cadena de conexiones que marcan la tendencia de dicho nodo, ofreciendo datos sobre el uso que hace la comunidad científica sobre la misma. Para ello se estableció unos protocolos de análisis: a) Unidad de análisis: palabras claves autores, palabras clave WoS; b) Umbral de frecuencia: P1 (10), P2 (29), P3 (29); c) Tipo de red: Co-ocurrencia; d) Umbral valor unión co-ocurrencia: P1 (6), P2 (7), P3 (7); e) Medida de normalización: Índice que equivalencia; f) Algoritmo de clustering: tamaño mínimo (12), tamaño máximo (6); g) Medida evolutiva: Índice de Jaccard; h) Medida superpuesta: Índice de inclusión.

\section{Resultados}

Los resultados que se presentan a continuación muestran por un lado el análisis cualitativo llevado a cabo en las 25958 referencias analizadas, y por otro lado el análisis de co-palabras de la producción científica.

Tal y como se observa en la tabla 1, la evolución de la producción científica ha ido creciendo desde 2009 hasta la actualidad. El análisis de la producción diacrónica muestra un repunte desde el año 2015 en adelante, recogiéndose el mayor pico de producción en el año 2018. Dentro de la temática de estudio, la búsqueda combinada de DIDE ofrece resultados mucho mayores, un total de 15957 producciones, sobre las otras dos combinaciones establecidas. Los resultados recogidos en la tabla 3 muestran que la temática está en fase de crecimiento exponencial, siendo una temática de interés para la comunidad científica.

\section{Tabla 1}

Producción diacrónica (2009-2019).

\begin{tabular}{llllll}
\hline & DIDE & DICL & INTE & Total & \% de 25958 \\
\hline $\mathbf{2 0 1 9}$ & 785 & 424 & 289 & 1498 & 5.77 \\
$\mathbf{2 0 1 8}$ & 2115 & 913 & 650 & 3678 & 14.77 \\
$\mathbf{2 0 1 7}$ & 2179 & 839 & 590 & 3608 & 13.90 \\
$\mathbf{2 0 1 6}$ & 1953 & 708 & 493 & 3154 & 12.15 \\
$\mathbf{2 0 1 5}$ & 1708 & 656 & 456 & 2820 & 10.86 \\
$\mathbf{2 0 1 4}$ & 1482 & 474 & 356 & 2312 & 8.91 \\
$\mathbf{2 0 1 3}$ & 1383 & 482 & 318 & 2183 & 8.41 \\
\hline
\end{tabular}

Fuente: Elaboración propia 
Tabla 1 (Continuación)

Producción diacrónica (2009-2019).

\begin{tabular}{lccccc}
\hline & DIDE & DICL & INTE & Total & \% de 25958 \\
\hline $\mathbf{2 0 1 2}$ & 1252 & 446 & 280 & 1978 & 7.62 \\
$\mathbf{2 0 1 1}$ & 1139 & 335 & 265 & 1739 & 6.70 \\
$\mathbf{2 0 1 0}$ & 1076 & 323 & 233 & 1632 & 6.29 \\
$\mathbf{2 0 0 9}$ & 885 & 271 & 200 & 1356 & 5.22 \\
\hline
\end{tabular}

Fuente: Elaboración propia

Teniendo en cuenta la productividad geográfica, se muestra que seis países son los que presentan mayores niveles de producción, siendo estos Estados Unidos (DICE: 5663; DICL: 1963; INTE: 1464), seguido de Inglaterra (DICE: 1271; DICL: 651; INTE: 485), Australia (DICE: 1251; DICL: 468; INTE: 355), España (DICE: 1241; DICL: 526; INTE: 286), Canadá (DICE: 762; DICL: 402; INTE: 264), y Brasil (DICE: 664; DICL: 342; INTE: 132).

En relación a las instituciones con mayor producción científica, las 8 universidades con mayor producción científica son University of California System (DICE: 366; DICL: 188; INTE: 160), University of London (DICE: 226; DICL: 98; INTE: 137), University of North Carolina (DICE: 265; DICL: 79; INTE: 53), State University system of Florida (DICE: 250; DICL: 85; INTE: 56), University of Texas System (DICE: 190; DICL: 53; INTE: 59), Harvard University (DICE: 137; DICL: 79; INTE: 72), California State University System (DICE: 187; DICL: 48; INTE: 0) y University System of Georgia (DICE: 187; DICL: 0; INTE: 35), entre otras.

Teniendo presente los datos mostrados en la tabla 2, se puede determinar que doce son los autores que superan el criterio de inclusión, destacando Bowman por encima del resto, teniendo un total de 31 producciones científicas.

Tabla 2

Autores más productivos ( $x \geq 13)$.

\begin{tabular}{lllll}
\hline & DIDE & DICL & INTE & Total \\
\hline Bowman & 31 & - & - & 31 \\
Wang Y & - & 8 & 12 & 20 \\
Martín-Pastore & 10 & 9 & - & 19 \\
Wang J & - & 8 & 10 & 18 \\
Evans & 17 & - & - & 17 \\
González-Gil & 9 & 8 & - & 17 \\
\hline
\end{tabular}

Fuente: Elaboración propia 
Tabla 2 (Continuación)

Autores más productivos ( $x \geq 13)$.

\begin{tabular}{lcccc}
\hline & DIDE & DICL & INTE & Total \\
\hline Pascarella & 16 & - & - & 16 \\
Zonderman & 16 & - & - & 16 \\
Denson & 15 & - & - & 15 \\
Olivencia & 14 & - & - & 13 \\
Keddie & 13 & - & - & 13 \\
Lee J. & 13 & - & - & 13 \\
\hline
\end{tabular}

Fuente: Elaboración propia

En relación a la productividad de fuente, específicamente en el tipo de producción, los artículos son los más usuales (DICE: 11856; DICL: 4262; INTE: 3330), seguidos de las comunicaciones (DICE: 2038; DICL: 370; INTE: 193), las revisiones bibliográficas (DICE: 508; DICL: 612; INTE: 598) y los capítulos de libros (DICE: 852; DICL: 199; INTE: 109), entre otros.

Con respecto a la fuente de los textos (tabla 3), el núcleo de producción no se acumula en una revista en concreto, sino más bien su distribución es equitativa. Entre todas las fuentes sobre la temática destaca Plos One.

\section{Tabla 3}

Núcleo de la producción científica $(x \geq 30)$.

\begin{tabular}{lccccc}
\hline & DIDE & DICL & INTE & Total & \% de 25958 \\
\hline Plos One & 70 & 105 & 108 & 283 & 1.09 \\
$\begin{array}{l}\text { Procedia Social and Behavioral } \\
\begin{array}{l}\text { Sciences } \\
\text { Edulearn Proceedings }\end{array}\end{array}$ & 170 & 25 & - & 195 & 0.75 \\
INTED Proceedings & 157 & 16 & - & 173 & 0.67 \\
$\begin{array}{l}\text { International Journal of Inclusive } \\
\text { Education }\end{array}$ & 144 & 26 & - & 170 & 0.65 \\
Academic Medicine & 82 & 48 & - & 130 & 0.5 \\
Revista de Educación Inclusiva & 81 & 29 & - & 110 & 0.42 \\
& 73 & 36 & - & 109 & 0.42 \\
\hline
\end{tabular}

Fuente: Elaboración propia 
Tabla 3 (Continuación)

Núcleo de la producción científica $(x \geq 30)$.

\begin{tabular}{llllll}
\hline & DIDE & DICL & INTE & Total & \% de 25958 \\
\hline ICERI Proceedings & 92 & 17 & - & 109 & 0.42 \\
$\begin{array}{l}\text { Advances in Social Science Education } \\
\text { and Humanities Research }\end{array}$ & 105 & - & - & 105 & 0.4 \\
$\begin{array}{l}\text { Teaching and Teacher Education } \\
\text { Equality Diversity and Inclusion }\end{array}$ & 83 & - & - & 83 & 0.32 \\
$\begin{array}{l}\text { Cocharne Database of Systematic } \\
\text { Reviews }\end{array}$ & - & 63 & - & 63 & 0.24 \\
\hline
\end{tabular}

Fuente: Elaboración propia

La comunidad científica escoge el inglés (DICE: 13779; DICL: 5286; INTE: 3810) como idioma principal para presentar sus resultados en los diferentes tipos de fuentes de producción, seguidos del español (DICE: 1140; DICL: 354; INTE: 190), portugués (DICE: 515; DICL: 127; INTE: 54), francés (DICE: 100; DICL: 25; INTE: 38), ruso (DICE: 107; DICL: 20; INTE: 5) y alemán (DICE: 81; DICL: 18; INTE: 14), entre otros.

Las investigaciones analizadas provienen principalmente del área Education Educational Research (DICE: 8336; DICL: 1112; INTE: 426), seguida de Psychology (DICE: 903; DICL: 338; INTE: 396), Linguistics (DICE: 519; DICL: 1112; INTE: 0), Business Economics (DICE: 843; DICL: 500; INTE: 125), Social Science Other Topics (DICE: 936; DICL: 254; INTE: 95), y Public Environmental Occupational Health (DICE: 529; DICL: 156; INTE: 326), entre otros.

Si se atiende a los documentos con más números de citas, destaca la obra desarrollada por Gay (2010), que tiene un total de 127 citas. Este manuscrito hace referencia a la formación docente para la diversidad, la cual se menciona con frecuencia pero no se desarrolla con suficiente detalle, lo que conlleva a actitudes y creencias no adecuadas de los docentes y discentes sobre las diferencias raciales, culturales y étnicas. El siguiente documento con más números de citas es el de Flores y Rosa (2015), con un total de 114 citas, el cual se centra en la crítica de los enfoques basados en la adecuación a la diversidad lingüística en la educación.

Centrando el estudio en el desarrollo estructural y temático de la producción científica, se puede observar una evolución continua y cambiante en el tiempo de las palabras claves utilizadas por los investigadores en el estudio de la diversidad en el ámbito educativo. En el primer periodo, comprendido entre los años 2009 y 2012, el número de palabras clave es de 17936. La evolución del primer al segundo periodo supone la salida de 11438 palabras clave y la introducción de 22666 nuevas palabras clave, habiendo una coincidencia entre periodos del 36\%, es decir, de 6498 palabras clave. En el segundo periodo las palabras claves utilizadas por los investigadores son 29164. Analizando la evolución de este segundo periodo hacia el tercer periodo, se observa una tendencia semejante entre el primero y segundo periodo, saliendo 20672 palabras clave e incluyéndose 17906 nuevas palabras clave en el tercer periodo. La coincidencia entre el segundo y el tercer periodo es del 32\%, es decir, 8492 palabras clave. El total de palabras clave en el tercer periodo es de 26398, tal y como se puede observar en la figura 1. 


\section{Figura 1}

Continuidad de palabras clave entre periodos contiguos

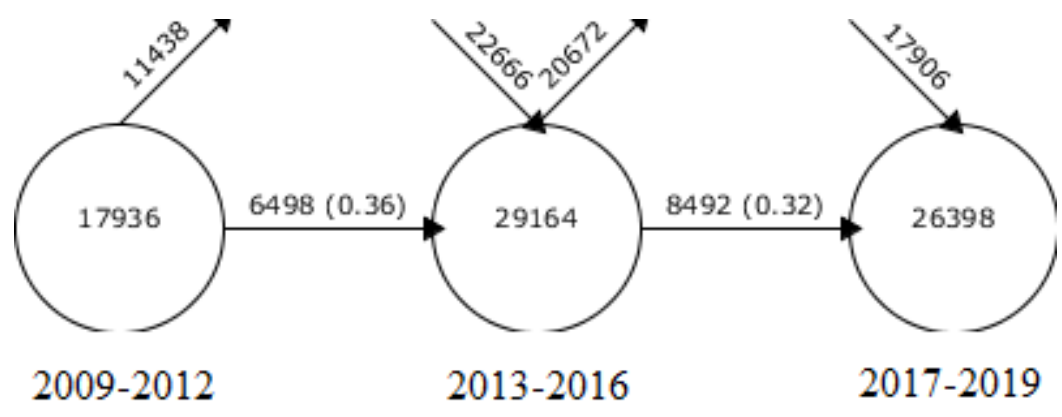

Fuente: Elaboración propia

Continuando con el análisis de las temáticas de investigaciones en los tres periodos establecidos, se muestra como en todos ellos la temática con más valor bibliométrico es DIVERSITY, habiendo una distancia considerable con el resto de temáticas, tal y como se muestra en la tabla 4.

\section{Tabla 4}

Rendimiento de la temática diversidad.

\section{Periodo 2009-2012}

\begin{tabular}{lllllll}
\hline & Obras & Índice-h & Índice-g & Índice-hg & Índice-q2 & Citas \\
\hline Diversity & 686 & 44 & 66 & 53.89 & 53.89 & 9773 \\
Intervention & 96 & 24 & 49 & 34.29 & 33.23 & 2710 \\
School & 76 & 20 & 38 & 27.57 & 30.66 & 1592 \\
Impact & 120 & 29 & 47 & 36.92 & 40.3 & 2945 \\
Gender & 50 & 18 & 30 & 23.24 & 24 & 995 \\
Healt-care & 31 & 12 & 19 & 15.1 & 17.32 & 408 \\
\end{tabular}

Periodo 2013-2016

\begin{tabular}{lcccccc}
\hline & Obras & Índice-h & Índice-g & Índice-hg & Índice-q2 & Citas \\
\hline Diversity & 1450 & 35 & 54 & 43.47 & 44.67 & 10091 \\
Prevalence & 97 & 21 & 39 & 28.62 & 25.92 & 1877 \\
Program & 125 & 19 & 30 & 23.87 & 23.47 & 1336 \\
\hline
\end{tabular}

Fuente: Elaboración propia 
Tabla 4 (Continuación)

Rendimiento de la temática diversidad.

Periodo 2013-2016

\begin{tabular}{lcccccc}
\hline & Obras & Índice-h & Índice-g & Índice-hg & Índice-q2 & Citas \\
\hline Program & 125 & 19 & 30 & 23.87 & 23.47 & 1336 \\
Children & 124 & 19 & 38 & 26.87 & 23.47 & 1844 \\
Performance & 115 & 16 & 25 & 20 & 20.78 & 1025 \\
Attitudes & 115 & 27 & 31 & 22.96 & 22.58 & 1332 \\
Cultural-diversity & 115 & 10 & 14 & 11.83 & 11.4 & 439 \\
Policy & 61 & 13 & 31 & 20.07 & 19.42 & 1052 \\
Women & 48 & 14 & 18 & 15.87 & 16.73 & 436 \\
\hline
\end{tabular}

Periodo 2017-2019

\begin{tabular}{lllllll}
\hline & Obras & Índice-h & Índice-g & Índice-hg & Índice-q2 & Citas \\
\hline Diversity & 1446 & 14 & 20 & 16.73 & 16.73 & 1863 \\
Undernutrition & 193 & 8 & 10 & 8.94 & 10.2 & 360 \\
Care & 95 & 5 & 6 & 5.48 & 5.48 & 103 \\
Attitudes & 171 & 7 & 9 & 7.94 & 7.48 & 244 \\
Women & 130 & 6 & 10 & 7.75 & 8.83 & 223 \\
Performance & 171 & 6 & 9 & 7.35 & 8.12 & 213 \\
School & 149 & 7 & 11 & 8.77 & 8.77 & 241 \\
Perspective & 58 & 5 & 5 & 5 & 5.48 & 69 \\
Obesity & 50 & 6 & 8 & 6.93 & 6.93 & 130 \\
Prevalence & 35 & 3 & 5 & 3.87 & 3.46 & 57 \\
Cultural-diversity & 49 & 3 & 4 & 3.46 & 3.46 & 27 \\
\hline
\end{tabular}

Fuente: Elaboración propia

Atendiendo a las diversas temáticas en el diagrama de la figura 2, se muestra que la temática DIVERSITY es el único tema motor en este periodo. La temática INTERVENTION está situada 
entre tema motor y tema muy desarrollado y aislado. Algo parecido ocurre con IMPACT, donde se sitúa entre tema motor y tema básico y transversal. La temática SCHOOL está como tema básico y transversal, y GENDER como temática incógnita, dado que no se sabe si está en desaparición o tiende a ser tema motor. En el periodo comprendido entre los años 2009 y 2012, DIVERSITY es la temática más citada (Figura 2).

\section{Figura 2}

Diagrama estratégico por citas para el periodo 2009-2012

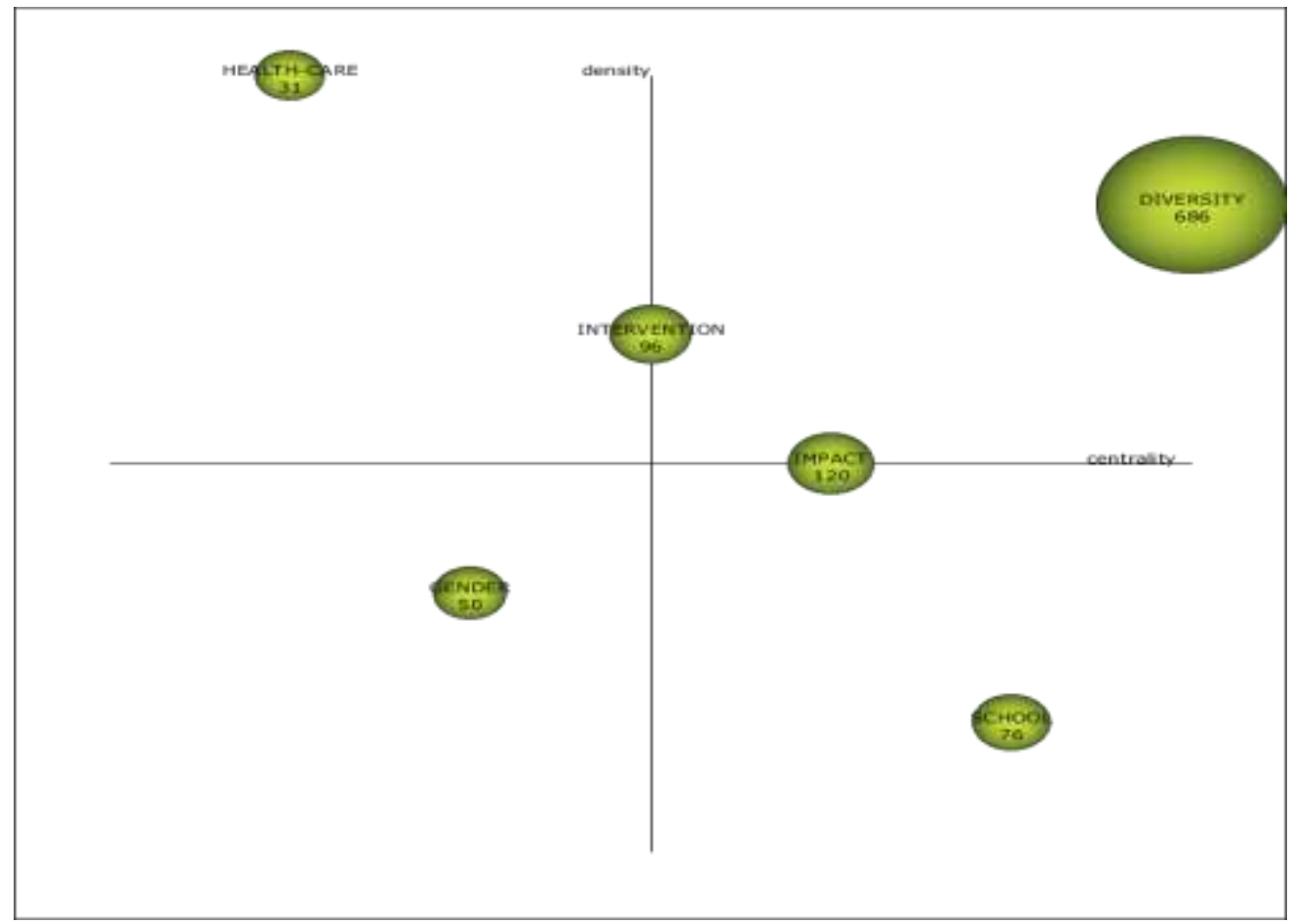

Fuente: Elaboración propia

En el diagrama estratégico del periodo comprendido entre 2013 y 2016 se observa, en comparación con el diagrama anterior, que aparecen más temas motores, tales como DIVERSITY, PROGRAM, CHILDREN y ATTITUDES. La temática PERFORMANCE se encuentra como tema básico y transversal, la temática PREVALENCE como temática muy desarrollada y aislada, y la temática WOMEN y CULTURAL DIVERSITY como temáticas incógnitas, dado que no se sabe si van a desaparecer o van a ser futuros temas motores en el ámbito científico. La temática con mayor citación vuelve a ser DIVERSITY (figura 3). 


\section{Figura 3}

Diagrama estratégico por citas para el periodo 2013-2016

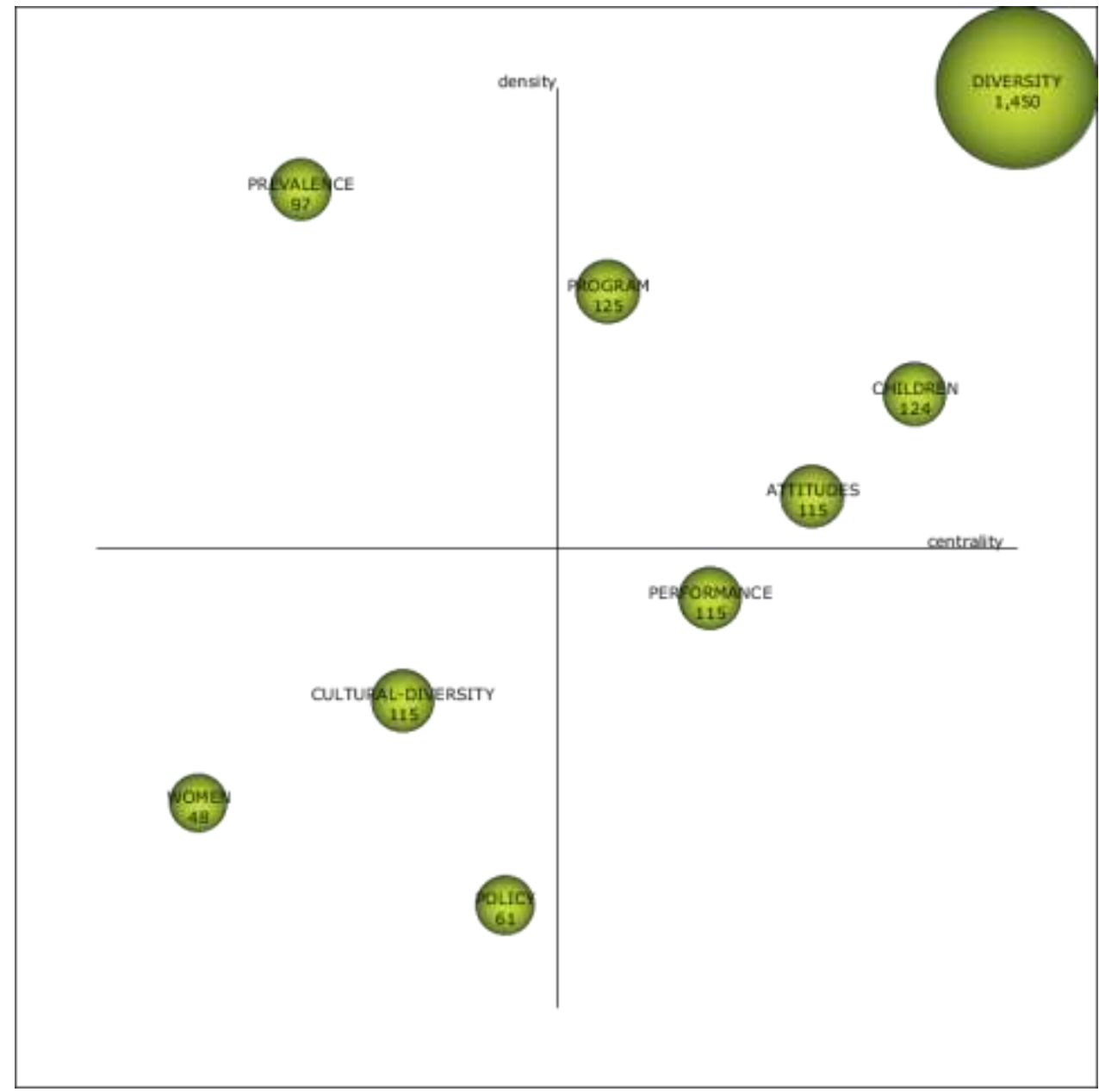

Fuente: Elaboración propia

El diagrama establecido en este periodo muestra las temáticas principales, siendo motores en este periodo son UNDERNUTRITION, DIVERSITY, WOMEN y ATTITUDES. EI resto de las temáticas se distribuye de forma uniforme por los distintos sectores. PERFORMANCE y SCHOOL son temáticas básica y transversal. OBESITY y CARE son temáticas muy desarrolladas y aisladas. PREVALENCE, CULTURAL DIVERSITY y PERSPECTIVE son temáticas incógnitas, dado que no se saben si van a ser futuros temas motores o van a tender a desaparecer, tal y como se muestra en la figura 4. 


\section{Figura 4}

Diagrama estratégico por citas para el periodo 2017-2019

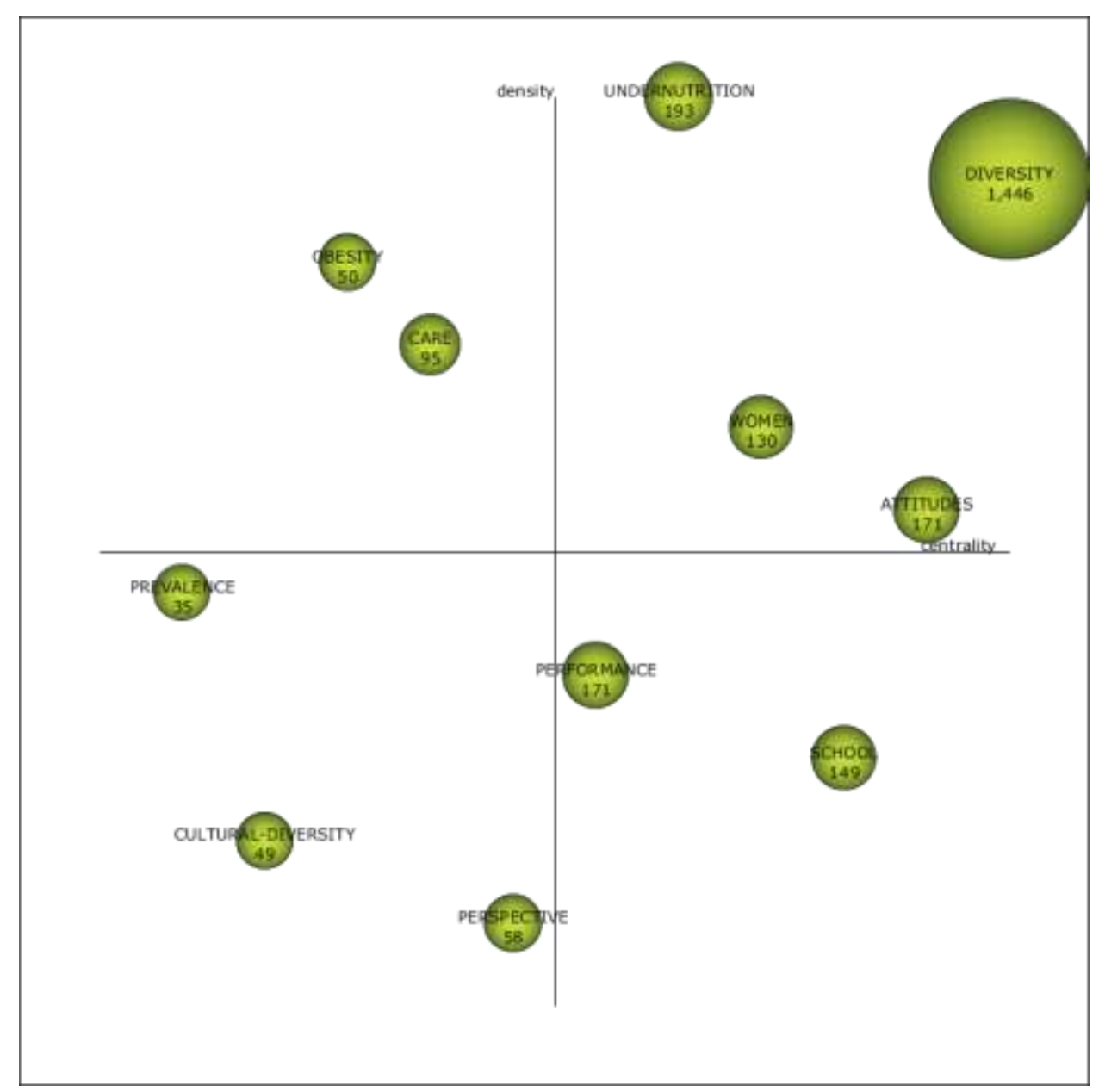

Fuente: Elaboración propia

Centrando el análisis ahora en la evolución científica del campo de estudio sobre la diversidad en el ámbito educativo en los tres periodos establecidos (2009-2012, 2013-2016 y 2017-2019) se muestra una continuidad temática muy afianzada y pronunciada, centrada principalmente en DIVERSITY. Las conexiones entre los periodos y temáticas también se muestran sólidas y con una tendencia clara y desarrollada. Del segundo al tercer periodo, se presenta más continuidad temática que entre el primero y segundo periodo, lo que muestra que desde el periodo 2013 en adelante la comunidad científica presenta una clara tendencia.

En el primero periodo, DIVERSITY es la temática con mayor índice-h. Es la única temática en este periodo que mantiene continuidad con el segundo periodo, además es la que mayor conexión conceptual muestra entre las diversas temáticas de este periodo.

En el segundo periodo se visualiza que la temática DIVERSITY es el eje central y de conexión entre los periodos primero y tercero, marcando la evolución de la temática de estudio. En este segundo periodo se empieza a mostrar una evolución temática en diversos temas, no solamente en DIVERSITY, sino también en PREVALENCE, PERFORMANCE, ATTITUDES, CULTURAL DIVERSITY y WOMEN. Las conexiones conceptuales con mayor fuerza son las establecidas entre DIVERSITY-DIVERSITY, PREVALENCE-PREVALENCE, ATTITUDES-ATTITUDES y CULTURAL DIVERSITY- CULTURAL DIVERSITY. Esto muestra que la base de estudio entre la población 
científica se está empezando a asentar en estas temáticas en los dos últimos periodos, tal y como se muestra en la figura 5.

\section{Figura 5}

Evolución temática por índice- $h$

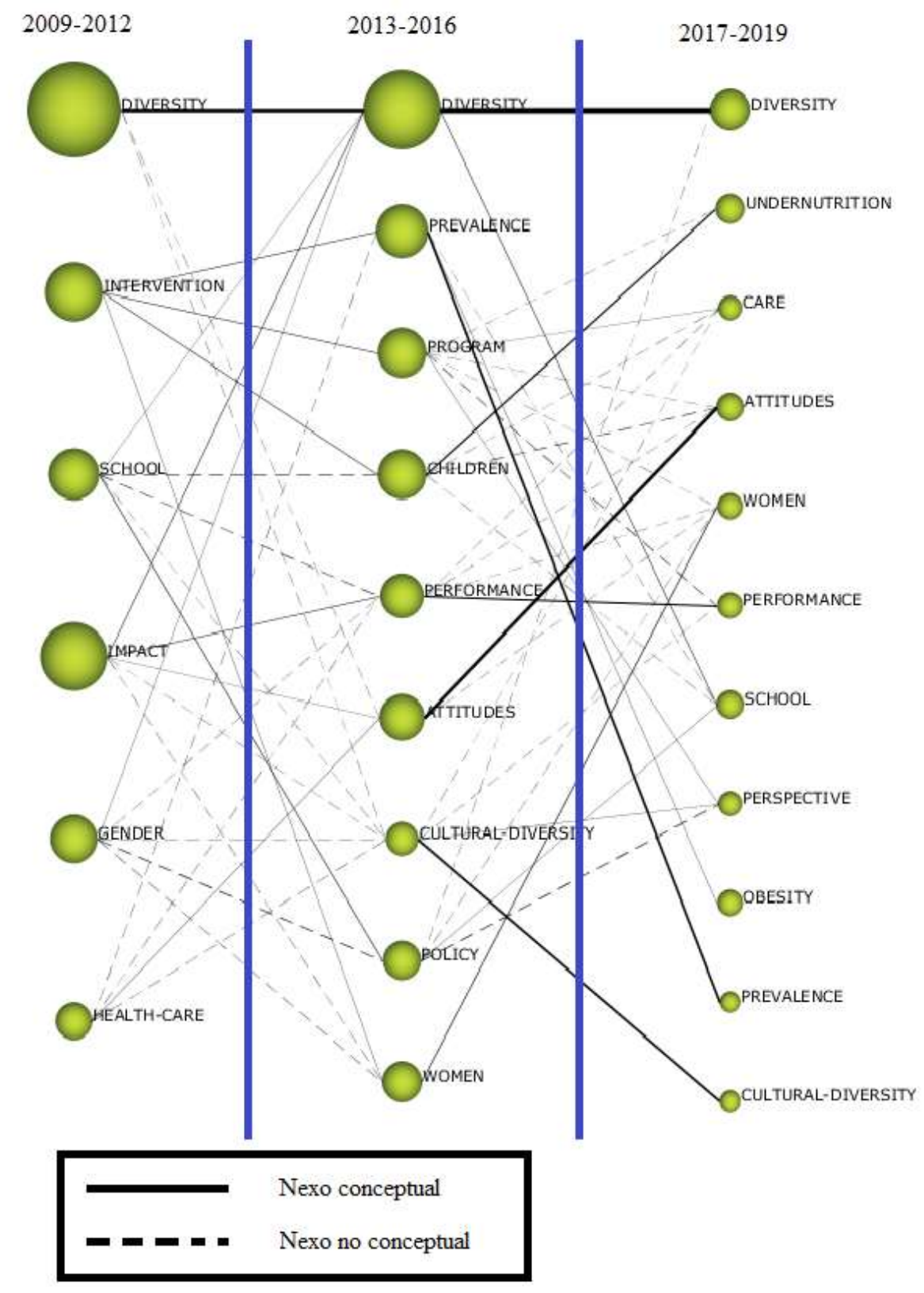

Fuente: Elaboración propia 


\section{Conclusiones y Discusión}

La diversidad en el ámbito educativo es un concepto que abarca un amplio espectro de temáticas. Esto se debe al carácter intrínseco y extrínseco del concepto, el cual abarca aspectos propios del ser humano, asociado a los aspectos que su propio entorno. En el ámbito educativo, mediante la aplicación de diversas normativa, trata de adecuar y dar respuestas a estas necesidades, mediante la aplicación de diversos programas de intervención, los cuales se centran en cambios a nivel metodológico, para poder desarrollar una educación inclusiva, o en medidas sociales que permitan atender las necesidades existentes en el estudiante (Airton y Koecher, 2019; Barbato y Turri, 2019; De Araujo et al., 2019; Jurado et al., 2020; Leseth y Engelsrud, 2019; Preece, 2019; Riediger et al., 2019; Zhuwao et al., 2019).

Los análisis aplicados muestran un patrón de crecimiento exponencial en los últimos años, sobre todo desde el año 2015 en adelante, donde la muestra crece considerablemente, produciéndose su pico más alto en el año 2018.

Se observa que la mayor producción científica, según establece la base de datos Web of Science, procede de investigadores de Estados Unidos, estando bastante distanciado con respecto de países como Inglaterra, Australia, España y Canadá, donde la temática de estudio también es de interés. Así mismo, la institución que más producción científica genera es la University of California System, seguida a corta distancia de instituciones como University of London, University of North Carolina o State University System of Florida.

Entre los autores que más producen sobre la temática, se encuentra Bowman, que muestra una carga de investigaciones bastante alta. Existe una diferencia de más de 10 producciones con respecto al segundo autor más prolífico en este campo de estudio. Los propios científicos utilizan los artículos escritos en inglés para mostrar sus resultados a la comunidad científica. Se puede observar que el título que más trabajo recopila al respecto es Plos One.

El área de investigación donde más se recogen estos estudios es Education Educational Research, destacando por encima del resto. Entre los autores más citados se encuentran Gay (2010), y Flores y Rosa (2015), siendo sus trabajos los referentes en el campo de la diversidad en el ámbito educativo.

Después de aplicar el mapeo conceptual en la temática de estudio, se obtienen las siguientes conclusiones:

- DIVERSITY: Esta temática puede considerarse el tema estrella en el estudio relacionado con la diversidad en el ámbito educativo. Aparece en los tres periodos como tema con mayor número de citas e índice-h. Durante el primer periodo, los autores centraban sus estudios en la educación superior, en la equidad, la justicia social, los estudiantes, la identidad, la inclusión, la educación, el multiculturalismo y la formación docente. En el segundo y tercer periodo sigue la misma tendencia que en el primero, añadiendo el género, el impacto y la experiencia al ámbito de investigación. Ello muestra que la base de estudios sobre diversidad lleva asentada en estos últimos años. Dada su evolución temática, se puede determinar que es un tema en auge y de gran interés para la comunidad científica.

- INTERVENTION: Esta temática solamente aparece en el primer periodo, como tema situado entre motor y en desarrollo y aislado. Los estudios se han centrado en el uso del vocabulario, el lenguaje, la prevención, los chicos jóvenes, las mujeres, los 
resultados y el comportamiento. Dada su evolución temática, se puede determinar que es un tema en decadencia.

- IMPACT: Aparece únicamente en el primer periodo, estando situado entre tema motor y tema básico y transversal. Los estudios sobre esta temática se centran en el colegio, la diversidad racial, la experiencia, las actitudes, las actuaciones, la percepción, los beneficios y los modelos. Dada su evolución temática, se puede determinar que es un tema en decadencia.

- SCHOOL: Surge tanto en el primer periodo como en el tercero, desapareciendo en el segundo, siendo este un claro ejemplo de brecha conceptual. En ambos periodos aparece como tema básico y transversal. Los estudios de esta temática están relacionados con el currículo, la participación, la educación médica, la preparación de los docentes, la familia, la política, los padres, la pedagogía y la educación multicultural. Dada su evolución temática, se puede determinar que es un tema en decadencia.

- GENDER: Esta temática aparece solo en el primer periodo como tema emergente o en desaparición. Los estudios sobre esta temática se centran en las diferencias, la sexualidad, el trabajo, la desigualdad, la ciencia y la etnicidad. Dada su evolución temática, se puede considerar un tema en decadencia.

- HEALTH CARE: Surge en el primer periodo como tema desarrollado y aislado. Los estudios sobre esta temática se centran en la competencia cultural, la disparidad, la comunicación y la psicología. Dada su evolución temática, se puede considerar que es un tema en decadencia.

- PREVALENCE: Aparece en el segundo periodo, como tema desarrollado y aislado, y en el tercero como tema emergente o en desaparición. Los estudios en el segundo periodo se centran en la obesidad, la diversidad genética, los adolescentes, los adultos, los factores de riesgo y la depresión. En el tercer periodo la perspectiva de estudio cambia, centrándose en la identificación y en las tendencias. Dada su evolución temática, no se puede determinar qué influencia va a tener en la comunidad científica, dado que actualmente es una incógnita.

- PROGRAM: Aparece únicamente en el segundo periodo como tema motor. Los estudios se centran en la implementación, los sucesos, la retención, la intervención, las barreras, las personas y los resultados. Dada su evolución temática, es un tema en decadencia.

- CHILDREN: Le ocurre exactamente igual que al anterior tema, dado que surge en el segundo periodo como tema motor, desapareciendo en el tercero. Los estudios se centran en la nutrición, la salud mental, la salud, la dieta, familia, la edad, los padres, los jóvenes, el comportamiento y el alfabetismo. Dada su evolución temática, es un tema en decadencia.

- ATTITUDES: Surge en el segundo periodo y se mantiene en el tercero. En ambos periodos aparece como tema motor. Tanto en el primer como en el segundo periodo los estudios se han centrado en la internacionalización de los estudiantes, el cuidado de la salud, la formación docente, la educación inclusiva, la discriminación, los conocimientos, los prejuicios, los inmigrantes, los estereotipos y las creencias. Dada su evolución temática, se puede determinar que es un tema en auge y de gran interés para la comunidad científica.

- PERFORMANCE: Aparece en el segundo y tercer periodo, como tema básico y transversal. Los estudios en este periodo se han centrado en las amenazas, las 
acciones afirmativas, la educación médica, la administración, la percepción, los conflictos, la comunicación, el trabajo, la organización, las habilidades y los logros. En el tercer periodo la base de estudios cambia completamente, centrándose en los estudiantes de centros educativos, los modelos, los equipos, el lugar de trabajo, los resultados y la diversidad en la administración. Dada su evolución temática, se puede determinar que es un tema en decadencia.

- CULTURAL DIVERISTY: Surge en el segundo y en el tercer periodo como temática incógnita, dado que puede ser considerado emergente o que tiende a desaparecer. En el primer periodo los estudios se centran en la diversidad lingüística, la autoeficacia, las competencias culturales, la migración, la perspectiva, los modelos, la educación multicultural, la educación intercultural, la inmigración, la multiculturalidad y la etnicidad. En el tercer periodo el número de temas de estudio se reduce, centrándose en el multiculturalismo, las competencias, la educación multicultural, la interculturalidad y la educación intercultural. Dada su evolución temática, no se puede determinar su evolución en los próximos años, dado que es una incógnita su influencia en la comunidad científica.

- POLICY: Aparece nada más en el segundo periodo, como tema emergente o en desaparición. La temática de estudio se centra en la comunidad, la ciencia, el currículo, los marcos de referencia y en la ciudadanía. Dada su evolución temática, se puede determinar que es un tema en decadencia.

- WOMEN: Surge en el segundo periodo como tema emergente o en desaparición, transformándose en el tercer periodo como tema motor. En el segundo periodo las investigaciones se centran en la interseccionalidad, los líderes, las minorías, la calidad y los compañeros de trabajo. Dada su evolución temática, se puede determinar que es un tema en auge y de gran interés para la comunidad científica.

- UNDERNUTRITION: Surge en el tercer periodo como tema motor. Los estudios se centran en los determinantes, la intervención, los niños, la nutrición, la salud, la diversidad en la dieta y en el estatus nutritivo. Dada su situación en el último periodo, se puede determinar que es un tema en auge y de gran interés para la comunidad científica.

- CARE: Aparece en el tercer periodo como tema desarrollado y aislado. Los estudios se centran los aspectos psicológicos, las búsquedas cualitativas, la educación médica, la competencia cultural, los accesos, los servicios, los padres y la salud mental. Dada su situación en el último periodo, se puede establecer que un tema en decadencia.

- OBESITY: Esta temática se encuentra en la misma situación que el anterior, dado que aparece nada más en el tercer periodo como tema desarrollado y aislado. Los estudios se centran en la dieta, la asociación, las enfermedades, la inclusión y en la actividad física. Dada su situación en el último periodo, se puede establecer que es un tema en decadencia.

- PERSPECTIVE: Surge en el tercer periodo como temática incógnita, dado que no se sabe si va a desaparecer o va a convertirse en tema motor. Los estudios se centran en los cambios, la motivación, los programas, la cultural, las estrategias, el marco de trabajo y en el soporte. Dada su situación en el último periodo, no se puede determinar su evolución en los próximos años.

Tal y como se puede observar, la temática DIVERSIDAD es la base de los estudios sobre diversidad en el ámbito educativo, centrado en aspectos relacionados con la equidad, la justicia social, los estudiantes, la identidad, la inclusión, la educación, el multiculturalismo y el género, aunque también está cogiendo fuerza estudios relacionados con las actitudes, las mujeres y la 
desnutrición, siendo cada vez más relevantes y abundantes las investigaciones sobre esta temática.

Se puede concluir que la base de estudio de la diversidad en el ámbito educativo es, en sí, la propia diversidad. Esta temática es el eje central de toda la producción científica, aunque su evolución ha generado cambios de perspectivas en la misma. Sus inicios se han centrado en la intervención y en el género, para luego avanzar e incluir nuevos aspectos en el campo de la investigación, como son los niños, la organización de la escuela, la nutrición y las actitudes de las personas. Por ello, el presente estudio bibliométrico pretende dar una visión a la comunidad científica de cara a las últimas tendencias y líneas de investigación de la atención a la diversidad en el campo educativo y de la orientación.

Las principales limitaciones encontradas en el desarrollo de este estudio ha sido depurar la base de datos, por la cantidad de referencias acumuladas y por haber temáticas que no eran propias del estudio planteado. Además, los resultados alcanzados en esta investigación parten de los criterios de inclusión y exclusión establecidos por los propios autores. También, se debe tener presente que la base de datos Web of Science se centra en producciones del mundo anglosajón, por lo que puede haber estudios que sean de relevancia científica y no se encuentren recogidos en esta investigación.

Como propuestas de mejora, se puede establecer el mismo estudio para otras bases de datos, ya sea Scopus o Google Académico, dado que la base de datos utilizada se focaliza en el ámbito anglosajón principalmente.

\section{Referencias bibliográficas}

Airton, L., y Koecher, A. (2019). How to hit a moving target: 35 years of gender and sexual diversity in teacher education. Teaching and teacher education, 80, 190-204. https://doi.org/10.1016/j.tate.2018.11.004

Álvarez, G. (2015). La educación comparada más allá de la REEC: análisis bibliométrico de la disciplina en las cinco revistas españolas afines de más impacto entre 1995 y 2014. Revista Española de Educación Comparada, 25, 19-45. https://doi.org/10.5944/reec.25.2015.14782

Amer, J., y Mir, A. (2017). Los procesos de implementación de los programas de mejora del aprendizaje en el rendimiento (PMAR). Perspectivas del profesorado, el alumnado y las familias. Revista Española de Orientación y Psicopedagogía, 28(3), 133-150. https://doi.org/10.5944/reop.vol.28.num.3.2017.21623

Azorín, C.M. (2017). Analysis of instruments on inclusive education and attention to diversity. Revista Complutense de Educación, 28(4), 1043-1060. https://doi.org/10.5209/RCED.51343

Barbato, G., y Turri, M. (2019). What do positioning paths of universities tell about the diversity of higher education systems? An exploratory study. Studies in Higher Education, 1-14. https://doi.org/10.1080/03075079.2019.1619681 
Byrd, D. (2019). The Diversity Distraction: A Critical Comparative Analysis of Discourse in Higher Education Scholarship. Review of Higher Education, 42, 135-172. https://doi.org/10.1353/rhe.2019.0048

Callon, M., Courtial, J.P., y Laville, F. (1991). Co-word analysis as a tool for describing the network of interactions between basic and technological research: The case of polumerchemsitry. Sciencitometric, 22(1), 155-205. https://doi.org/10.1007/BF02019280

Castro, M.M., y Eirin, R. (2018). Evaluation of physical education materials for attention to diversity. Analysis of a proposal. Retos. Nuevas tendencias en educación física, deporte y recreación, (34), 356-362.

Cobo, M.J., López, A.G., Herrera, E., y Herrera, F. (2011). Science mapping software tools: Review, analysis, and cooperative study among tools. Journal of the American Society for Information Science and Technology, 62(7), 1382-1402. https://doi.org/10.1002/asi.21525

Cobo, M.J., López, A.G., Herrera, E., y Herrera, F. (2012). SciMAT: A new science mapping analysis software tool. Journal of American Society for Information Science and Technology, 63(8), 1609-1630. https://doi.org/10.1002/asi.22688

De Araujo, L.A., Cordeiro, A.P., y Mosca, C.R. (2019). A meeting with diversity in elementary school education trrough the Project "simplesmente diferente" from the perspective of the teacher, the child and the family. Revista Ibero-Americana de Estudos em Educação, 14, 775-790. https://doi.org/10.21723/riaee.v14iesp.1.12206

Elkins, M.R., Maher, C.G., Herbert R.D., Moseley, A.M., y Sherrington, C. (2010). Correlation between the journal impact factor and three other journal citation indices. Scientometrics, 85, 81-93. https://doi.org/10.1007/s11192-010-0262-0

Flores, N., y Rosa, J. (2015). Undoing Appropriateness: Raciolinguistic Ideologies and Language Diversity in Education. Harvard Educational Review, 85(2), 7-29.

Franceschet, M. (2010). Journal influence factors. Journal of Informetrics, 3(4), 239-248. https://doi.org/10.1016/j.joi.2009.12.002

Gay, G. (2010). Acting on Beliefs in Teacher Education for Cultural Diversity. Journal of Teacher Education, 61(1), 143-152. https://doi.org/10.1177/0022487109347320

Hinojo-Lucena, F.J., Dúo-Terrón, P., Ramos, M., Rodríguez-Jiménez, C., y Moreno-Guerrero, A.J. (2020). Scientific Performance and Mapping of the Term STEM in Education on the Web of Science. Sustainability, 12(6), 1-20. https://doi.org/10.3390/su12062279

Hirsch, J.E. (2005). An index to quantify an individual's scientific research output. Proceedings of National Academy of Sciences, 102(46), 16569-16572. https://doi.org/10.1073/pnas.0507655102

Hutto, B., Catalá, F., y Moher, D. (2016). La extensión de la declaración PRISMA para revisiones sistemáticas que incorporan metaanálisis en red: PRISMA-NMA. Medicina Clínica, 147(6), 262-266. https://doi.org/10.1016/j.medcli.2016.02.025

Jurado, P., Moreno-Guerrero, A.J., Marín-Marín, J.A., y Soler, R. (2020). The Term Equity in Education: A Literature Review with Scientific Mapping in Web of Science. International Journal of Environmental Research and Public Health, 17(10), 1-18. https://doi.org/10.3390/ijerph17103526

Ley Orgánica 2/2006, de 3 de mayo de Educación. Boletín Oficial del Estado, núm. 106, pp. 17158-17207. Texto consolidado. 
Leseth, A., y Engelsrud, G. (2019). Situating cultural diversity in movement. A case study on physical education teacher education in Norway. Sport Education and Society, 24(5), 468479. https://doi.org/10.1080/13573322.2017.1414694

López, E., Vázquez, E., y Sarasola, J.L. (2015). Estudio Bibliométrico Pixel-Bit. Revista de Medios y Educación (2000-2013). Pixel-Bit. Revista de Medios y Educación, 46, 65-85. https://doi.org/10.12795/pixelbit.2015.i46.05

Montero, J., Cobo, M.J., Gutiérrez, M., Segado, F., y Herrera, E. (2018). Mapeo científico de la Categoría "Comunicación" en WoS (1980-2013). Comunicar, 55(26), 81-91. https://doi.org/10.3916/C55-2018-08

Pegalajar, M.C. (2017). Attention to Diversity from the Perspective of Students Doing the Masters in Secondary Education at the University of Jaen. Educatio Siglo XXI, 35(1), 77-98. https://doi.org/10.6018/j/286231

Preece, S. (2019). Elite bilingual identities in higher education in the Anglophone world: the stratification of linguistic diversity and reproduction of socio-economic inequalities in the multilingual student population. Journal of Multilingual and Multicultural development, 40(5), 404-420. https://doi.org/10.1080/01434632.2018.1543692.

Quevedo, R., y López, W. (2010). Análisis bibliométrico de las revistas multidisciplinares de psicología incorporadas en la Web of Science (2008-2009). Psicología: Reflexao e Crítica, 23(2), 384-408.

Riediger, N.D., Kingson, O., Mudruj, A., Farquhar, K.L., Spence, K.A., Vagianos, K., y Suh, M. (2019). Diversity and Equity in Dietetics and Undergraduate Nutrition Education in Manitoba. Canadian Journal of Dietetic practice and research, 80(1), 44-46. https://doi.org/10.3148/cjdpr-2018-034

Rodríguez, A.M., Trujillo, J.M., y Sánchez, J. (2019). Impacto de la productividad científica sobre competencia digital de los futuros docentes: aproximación bibliométrica en Scopus y Web of Science. Revista Complutense de Educación, 30(2), 623-646.doi: 10.5209/RCED.58862

Rodríguez, A.M., Raso F., y Ruiz, J. (2019). Competencia digital, educación superior y formación del profesorado: un estudio de meta-análisis en la Web of Science. Pixel- Bit. Revista de Medios de Comunicación, 54, 65-81. https://doi.org/10.12795/pixelbit.2019.i54.04

Ruiz, R., Delgado, E., y Jiménez, E. (2010). Principios y criterios utilizados en España por la Comisión Nacional Evaluadora de la Actividad Investigadora (CNEAI) para la valoración de las publicaciones científicas: 1989-2009. Psicothema, 222, 898-908.

Sánchez, A.M., y García, A. (2017). Intercultural education. A project of attention to diversity from the performing arts. Education, Health and ICT for a Transcultural World, 237, 856-862. https://doi.org/10.1016/j.sbspro.2017.02.183

Sincer, I., Severiens, S., y Volman, M. (2019). Teaching diversity in citizenship education: Contextrelated teacher understandings and practices. Teaching and teacher education, 78, 183192. https://doi.org/10.1016/j.tate.2018.11.015

Tennial, R.E., Salomon, E.D., Hammonds-Odie, L., McDowell, G.S., Moore, M., Roca, A., y Marcette, J. (2019). Formation of the Inclusive Environments and Metrics in Biology Education and Research (iEMBER) Network: Building a Culture of Diversity, Equity, and Inclusion. CBE-LIFE Sciences Education, 18(1), 1-8. https://doi.org/10.1187/cbe.18-030042

Todeschini, R., y Baccini, A. (2016). Handbook of Bibliometric Indicators: Quantitative Tools for Studying and Evaluating Research. Wiley-VCH. 
Velasco, B., Eiros, J. M., Pinilla, J. M., y San Román, J. A. (2012). La utilización de indicadores bibliométricos para evaluar la actividad investigadora. Aula Abierta, 40(2), 75-84.

Veliz, L., Bianchetti, A.F., y Silva, M. (2019). Intercultural skills in primary health care: a challenge for higher education in contexts of cultural diversity. Cuadernos de Saúde Publica, 35(1), 111. https://doi.org/10.1590/0102-311X00120818

Zhuwao, S., Ngirande, H., Ndlovu, W., y Setati, S.T. (2019). Gender diversity, ethnic diversity and employee performance in a South African higher education institution. SA Journal of Human Resource Management, 17, 1-8. https://doi.org/10.4102/sajhrm.v17i0.1061

Fecha de entrada: 7 julio 2019

Fecha de revisión: 25 mayo 2020

Fecha de aceptación: 28 mayo 2020 